\title{
Peritonitis Of Unknown Origin In a Patient With Refractory Shock
}

\author{
E. Guerra Hernández ${ }^{1 *}$, Z. Hussein Dib González ${ }^{1}$ and R. Fariña Castro ${ }^{1}$ \\ ${ }^{1}$ Anesthesiology Service, Hospital Universitario de Gran Canaria Doctor Negrín, Las Palmas de Gran Canaria, Spain
}

Received: April 07 2018; Accepted: June 14 2018; Published: July 112018

*Corresponding author: Elisabet Guerra Hernández, Anesthesiology Service, Hospital Universitario de Gran Canaria Doctor Negrín, Las Palmas de Gran Canaria, Spain. E-mail: ecgh_7@hotmail.com

\begin{abstract}
Toxic Shock Syndrome (TSS) is an uncommon acute systemic disease, in which toxins that cause high morbidity and mortality are produced [1]. An early diagnosis, a rapid surgical debridement of the areas affected and appropriate antibiotics are the only ways to reduce the elevated mortality of this clinical condition [2]. Using this case, it is intended to stress the extreme gravity and importance of an early intervention of the disease, by attempting to limit the sequelae that could occur and thus being able to give a better quality of life to the patients that survive [2].
\end{abstract}

Keywords: Toxic Shock Syndrome (TSS); Streptococcus pyogenes;

\section{Case Report}

The case concerns a 36 year-old woman with no relevant medical history who had inserted a vaginal ring as a contraceptive method seven days before. At three days after this insertion, she started with a latent clinical picture of fever and abdominal pain, being diagnosed as urinary infection for which she was prescribed home treatment with ciprofloxacin with no obvious improvement.

Due to the persistency of the symptoms she arrived in the Emergency Department in a serious clinical condition with an arterial hypotension (that, at that time, required catecholamines at high doses of up to $3 \mathrm{ug} / \mathrm{kg} / \mathrm{min}$ of norepinephrine), anuria, confusion with a decrease in the level of consciousness (Glasgow Coma Scale 8), petechiae on the trunk, conjunctival icterus, tachypnoea at 22 breaths per minute, poor peripheral perfusion and vomiting. She was assessed by the general and gastrointestinal surgery department and given the acute abdomen picture and the suspicion of acute appendicitis, she was taken to surgery to perform an exploratory laparoscopy with the prior withdrawal of the vaginal ring. The laboratory results showed: $80,000 / \mathrm{L}$ white cells, 80,000/L platelets, creatinine $1.2 \mathrm{mg} / \mathrm{dL}$, Quick index of $44 \%$ and bilirubin $1.8 \mathrm{mg} / \mathrm{dL}$, with rest of no interest. The imaging tests (ultrasound and abdominal scan) were suggestive of acute appendicitis. With these data, at that time she obtained a Sequential Organ Failure Assessment (SOFA) score of +14 points.

General anaesthesia was performed for the procedure, with etomidate $14 \mathrm{mg}$, fentanyl 75 ug and intravenous succinylcholine
75 ug. Cormack I was observed in the orotracheal intubation. The anaesthesia was maintained with sevoflurane at 0.7-0.8 MAC (Minimum Alveolar Concentration) for a Bispectral index (BIS) between 45 and 60, fentanyl and cisatracurium $0.1-0.15 \mathrm{ug} / \mathrm{kg} /$ min intravenously for a Train-Of-Four (TOF) ratio of 1:4. During the surgery, only a turbid fluid was found in the pelvic area, which was sent for culture. On there not being a clinical diagnosis that could justify the poor health state of the patient, a Toxic Shock Syndrome (TSS) was suspected and empiric antibiotic treatment was started with imipenem, amikacin, linezolid and clindamycin until the results of the antibiogram were obtained. The patient was admitted to the intensive care unit in a critical condition and advanced life support measures were used due to the development of multi-organ dysfunction syndrome and haemodynamic instability, deterioration of renal function, with a need for Continuous Renal Replacement Therapy (CRRT), liver failure, respiratory failure with invasive mechanical ventilation, coagulopathy and conjunctival icterus.

From a microbiology perspective at 48 hours, Streptococcus pyogenes were isolated in the blood cultures and abdominal fluid, which confirmed the clinical suspicion for which it was decided to scale down the antibiotic prescription to imipenem and clindamycin. The patient showed a slight improvement and the SOFA score at that time was 9.

At 7 days, the progression was very favourable with the vasoactive amines being withdrawn along with the assisted ventilation and the CRRT. She was discharged from the intensive care unit on the 18th day of her admission, to the Internal Medicine hospital ward, where her progression was satisfactory and subsequently discharged home. She currently carries out daily activities with an excellent quality of life.

\section{Discussion}

TSS is an acute systemic disease caused by the production of superantigen toxins of Staphylococcus aureus and Streptococcus pyogenes (Group A Streptococcus (GAS)). It is a rare disease, with a high morbidity and mortality rate ( $>25 \%$ in the first 24 hours) [1]. 
The SOFA (Sequential Organ Failure Assessment) score calculated on admission of the patient and during the first days in Critical Care is a good indicator of the prognosis [3 \& 4]. Both the mean and the highest score are very useful predictors of the outcome. A SOFA score of 13-14 points on admission is associated with a mortality of $50-60 \%$. Independently of the initial score, an increase in the SOFA score in the first 48 hours of admission into Critical Care predicts a mortality rate of at least $50 \%$.

In this case, the SOFA score at 48 hours was less than the initial one, meaning a possible satisfactory outcome could be predicted. Due to the relative infrequency, the high morbidity and mortality, and the rapid progression of the symptoms, a high index of suspicion is required to make an early diagnosis and a rapid start of the appropriate treatments [5-7]. It is characterised by an acute, progressive clinical picture, together with a high temperature, arterial hypotension of sudden onset and multiorgan failure [8]. Occasionally, it presents with peritonitis with no apparent cause [9].

The clinical symptoms of fever, multisystemic involvement and shock together with the laboratory results are the basis of its diagnosis [2 \& 8]. [8]:

The diagnostic criteria of streptococcal TSS are the following

\section{Isolation of Group A beta-haemolytic Streptococcus:}

a) In normally sterile medium, such as blood, cerebrospinal or peritoneal fluid, tissue biopsy.

b) In non-sterile places such as the throat, vagina or sputum.

\section{Clinical signs of severity:}

a) Arterial hypotension (systolic blood pressure $\leq 90 \mathrm{mmHg}$ in adults).

b) Two or more of the following signs: renal failure with creatinine $>2 \mathrm{mg} / \mathrm{dL}$, coagulopathy with platelets $\leq 100,000 / \mathrm{L}$ or disseminated intravascular coagulation, liver failure with alanine aminotransferase, aspartate aminotransferase or total bilirubin two times higher than the upper limit of normal, adult respiratory distress syndrome, generalised rash, a macular rash that can present with desquamation, soft tissue necrosis, including necrotising fasciitis, myositis or gangrene.

This syndrome is classified as probable if criteria $1 \mathrm{~b}$ and 2 (a and b) are met, if there is no other cause that could produce the clinical picture of the patient and as definitive if criteria $1 \mathrm{a}$ and 2 (a and b) are met.

The process consists of making an early diagnosis, recovery measures with aggressive fluid replacement, respiratory and inotropic support. Surgery should be performed as soon as possible with surgical debridement of wounds, drainages or abscesses. As well an antibiotic treatment with the correct choice of empirical antibiotics, it should be considered the need to cover methicillin resistant Staphylococcus aureus, the use of adjuvant treatment with clindamycin and intravenous immunoglobulins, the review of the progress of the clinical picture with repeated surgery of the infection focus and the correct use and duration of the antibiotics [2]. Finally, it should be taken into account reducing the risk of new cases due to contact. And finally it should be stressed the need to reduce the risk of new cases due to contact.

\section{References}

1. Parks T, Barrett $L$ and Jones N. Invasive streptococcal disease: a review for clinicians. British medical bulletin.Br Med Bull . 2015;115(1):7789. Doi: $10.1093 / \mathrm{bmb} / \mathrm{ldv} 027$.

2. Wilkins AL, Steer AC, Smeesters PR and Curtis N. Toxic shock syndrome - the seven Rs of management and treatment. J Infect. 2017;74 Suppl:S147-S152. Doi: 10.1016/S0163-4453(17)30206-2.

3. Jones AE, Trzeciak S and Kline JA. The Sequential Organ Failure Assessment score for predicting outcome in patients with severe sepsis and evidence of hypoperfusion at the time of emergency department presentation. Crit Care Med. 2009;37(5):1649-1654. Doi: 10.1097/CCM.0b013e31819def97.

4. Vincent JL, Moreno R, Takala J, Willatts S, De Mendonça A and Bruining H. et al. The SOFA (Sepsis-related Organ Failure Assessment) score to describe organ dysfunction/failure. On behalf of the working group on Sepsis-Related Problems of the European Society of Intensive Care Medicine. Intensive Care Med. 1996;22(7):707-710.

5. Young K, Luni FK and Yoon Y. Toxic Shock Syndrome: an unusual organism. Am J Med Sci. 2016; 352 (1): 86-90. Doi: 10.1016/j. amjms.2016.04.002.

6. Low DE. Toxic shock syndome: major advances in pathogenesis, but not treatment. Crit Care Clin 2013; 29(3): 651-675. Doi: 10.1016/j. ccc.2013.03.012.

7. Wood TF, Potter MA and Jonasson O. Streptococcal toxic shock-like syndrome. The importance of surgical intervention. Ann Surg 1993; 217(2): 109-114.

8. Lappin E and Ferguson AJ. Gram-positive toxic shock syndromes. Lancet Infect dis. 2009;9(5):281-290. Doi: 10.1016/S14733099(09)70066-0.

9. Ramnik V Patel, Hemant Kumar, Bharat More and AShok Rajimwale. Primary group A streptococal septic shock syndrome simulating perforated appendicitis in a previously healthy girl. BMJ Case Rep. 2013;Doi:10.1136/bcr-2013-009502. 